\title{
Characterization of glucagon-like peptide 2 pathway member expression in bovine gastrointestinal tract
}

\author{
E. E. Connor, ${ }^{* 1}$ R. L. Baldwin VI, ${ }^{*}$ A. V. Capuco, ${ }^{*}$ C. M. Evock-Clover, ${ }^{*}$ S. E. Ellis, $†$ and K. S. Sciabicał \\ *Beltsville Agricultural Research Center, USDA-ARS, 10300 Baltimore Avenue, Beltsville, MD 20705 \\ †Animal and Veterinary Science Department, 129 Poole Agricultural Center, Clemson University, Clemson, SC 29634 \\ ‡Beckman Coulter Inc., 250 South Kraemer Boulevard, Brea, CA 92822
}

\section{ABSTRACT}

Glucagon-like peptide 2 (GLP-2), secreted by enteroendocrine cells, has several physiological effects on the intestine of monogastric species, including promotion of growth of intestinal epithelium, reduction of epithelial cell apoptosis, and enhancement of intestinal blood flow, nutrient absorption, and epithelial barrier function. The regulatory functions of GLP-2 in the ruminant gastrointestinal tract (GIT) have not been well studied. The objectives of this investigation were to characterize the mRNA expression of 4 members of the GLP-2 pathway throughout the bovine GIT, including (1) proglucagon $(G C G)$, the parent peptide from which GLP-2 is derived through cleavage by prohormone convertase; (2) prohormone convertase (PCSK1); (3) GLP-2 receptor $(G L P 2 R)$; and (4) dipeptidyl peptidase IV (DPP4), the enzyme that inactivates GLP-2. Gene expression was evaluated in rumen, reticulum, omasum, abomasum, duodenum, jejunum, ileum, cecum, and rectum collected at slaughter from prepubertal heifers, mature cows in early, mid, and late lactation, and nonlactating cows ( $\mathrm{n}=3$ per stage) by a gene expression profiling assay. In addition, mRNA expression of 14 genes involved in nutrient transport, enzyme activity, blood flow, apoptosis, and proliferation were evaluated in the 9 GIT tissues for their association with $G C G$ and GLP2R mRNA expression. Immunohistochemistry was used to localize GLP2R protein in tissues of the lower GIT. Results indicated that mRNA expression of $G C G, P C S K 1, G L P 2 R$, and DPP 4 varies across the 9 GIT tissues, with greatest expression in small and large intestines, and generally nondetectable levels in forestomachs. Expression of DPP4 and GLPQR mRNA varied by developmental stage or lactational state in intestinal tissues. Expression of $G C G$ or $G L P 2 R$ mRNA was correlated with molecular markers of proliferation, apoptosis, blood flow, enzyme activity, and urea transport, depending on the tissue examined, which suggests

Received February 25, 2010.

Accepted July 8, 2010.

${ }^{1}$ Corresponding author: erin.connor@ars.usda.gov a potential for involvement of GLP-2 in these physiological processes in the ruminant GIT. The GLP2R protein was expressed in intestinal crypts of the bovine GIT, which is consistent with the distribution in monogastric species. Our findings support a functional role of the GLP-2 pathway in bovine GIT and the potential for use of GLP-2 as a therapy to improve intestinal function and nutrient absorption in ruminants.

Key words: cattle, gastrointestinal tract, gene expression, glucagon-like peptide 2

\section{INTRODUCTION}

Glucagon-like peptide 2 (GLP-2) is a hormone secreted by intestinal L cells, which has been shown in monogastric species to increase cell proliferation in the intestinal crypts, reduce apoptosis of intestinal mucosa, suppress inflammation, and enhance nutrient absorption in the gut (Lovshin and Drucker, 2000; Burrin et al., 2003; Drucker, 2005). The peptide has been used to improve intestinal growth and integrity after injury or after regression resulting from parenteral nutrition in pigs, and may be a viable treatment to improve intestinal function in premature infants (Burrin et al., 2007), as well as adults with short-bowel syndrome (Jeppesen, 2003).

The biological actions of GLP-2 are determined by the liberation of the peptide from its parent protein proglucagon (GCG) via the enzyme prohormone convertase (PCSK1), its degradation by dipeptidyl peptidase IV (DPP4), and interaction with its $\mathrm{G}$ protein-coupled receptor, GLP-2 receptor (GLP2R). To date, functional studies of the GLP-2 pathway in livestock have been limited to swine, although preliminary reports indicate that $G C G$ and $G L P 2 R$ mRNA are expressed in bovine gastrointestinal tract (GIT; Taylor-Edwards et al., 2008), and that GLP-2 treatment enhances splanchnic blood flow and mass of the small intestine in calves (Taylor-Edwards et al., 2009a,b). The complete functions and therapeutic potential of GLP-2 in ruminants are unknown, but its use as a treatment for intestinal diseases such as calf scours has been suggested (Burrin et al., 2003). 
The purpose of this study was to evaluate mRNA expression of GCG, PCSK1,DPP4, and GLP2R, as well as mRNA for nutrient transporters, markers of apoptosis, proliferation, growth, enzyme activity, and blood flow in the bovine GIT. Gene expression was evaluated during 5 stages of development or lactation, including prepubertal heifers, lactating cows during early, mid, and late lactation, and nonlactating cows. A 22-gene multiplex expression assay was used to test the hypothesis that changes in lactational stage are associated with changes in expression of GLP-2 pathway members. In addition, we tested the hypothesis that expression of $G C G$ or GLP2R mRNA is correlated to changes in expression of mRNA for markers of apoptosis, proliferation, growth, enzyme activity, and blood flow, because GLP-2 is known to affect these processes in other species. Last, cellular expression of GLP2R protein was characterized in the lower bovine gut by immunohistochemistry.

\section{MATERIALS AND METHODS}

\section{Bovine Tissues}

Intestinal tissues including rumen, reticulum, omasum, abomasum, duodenum, jejunum, ileum, cecum, and rectum were collected at slaughter from culled adult Holstein cows from the Beltsville Agricultural Research Center dairy herd (Beltsville, MD) at the institutional abattoir, and from prepubertal Holstein heifers (100 to $130 \mathrm{~d}$ of age) from a study conducted at Clemson University (Clemson, SC). All animal procedures associated with the heifer samples were approved by the Clemson University Animal Care and Use Committee. For each developmental stage examined, gene expression was based on 3 animals. Among the lactating Holsteins, cows were categorized as early lactation [19 to $92 \mathrm{DIM} ; 3.7 \pm 1.4 \mathrm{yr}$ of age (mean $\pm \mathrm{SD}$ ); lactation number of $2.3 \pm 1.5$ (mean $\pm \mathrm{SD}$ )], mid lactation (138 to 201 DIM; $4.9 \pm 4.1$ yr of age; $3.0 \pm 3.5$ lactations), or late lactation (250 to $414 \mathrm{DIM} ; 4.9 \pm 2.1 \mathrm{yr}$ of age; $2.7 \pm 2.1$ lactations). Nonlactating cows were $5.3 \pm$ 2.1 yr of age, nonpregnant, and killed either 59 or $77 \mathrm{~d}$ after cessation of milking.

At slaughter, each tissue was dissected and rinsed in tap water (rumen, reticulum, omasum, and abomasum) or physiological saline (duodenum, jejunum, ileum, cecum, and rectum) to remove residual feed and GIT contents. In an attempt to maintain consistency for tissue sampling, rumen was sampled from the anterior portion beneath the reticulum and below the rumen fluid layer; abomasum was sampled from the lower, ventral surface of the posterior compartment; duodenum was sampled posterior to the bile ducts; ileum was sampled anterior to the ileo-cecal junction; cecum was sampled from the most distal region of the enclosed sack; and rectum was sampled anterior to the anus.

For RNA analysis, sampling was limited to the epithelial layers. For the small and large intestinal segments, a glass slide was used to scrape the epithelial mucosal layer for sample collection. Samples were then immediately immersed in RNAlater RNA stabilization solution (Ambion, Austin, TX) and stored at $4^{\circ} \mathrm{C}$ overnight. Excess RNAlater was then removed and preserved tissues were stored at $-80^{\circ} \mathrm{C}$ until RNA extraction. Whole tissues were also sampled for sectioning and subsequent immunohistochemical analysis. Tissue pieces were placed in glass scintillation vials with 15 $\mathrm{mL}$ of $10 \%$ neutral buffered formalin ( $\mathrm{pH} 6.8$ to 7.2 ; Richard-Allan Scientific, Kalamazoo, MI) and stored at $4^{\circ} \mathrm{C}$ overnight. Excess neutral buffered formalin was removed and tissue pieces were rinsed once with $70 \%$ ethanol, and then stored at $4^{\circ} \mathrm{C}$ in fresh $70 \%$ ethanol until paraffin embedding and tissue sectioning.

\section{RNA Extraction}

Frozen tissues were homogenized in Trizol reagent (Invitrogen, Carlsbad, CA), and total RNA was extracted using the RNeasy Midi Kit (Qiagen, Valencia, CA) with on-column DNase digestion. Quality of RNA was assessed using a 2100 Bioanalyzer and RNA 6000 Nano LabChip kits (Agilent Technologies, Palo Alto, $\mathrm{CA}$ ), and RNA concentration was determined using a NanoDrop ND-1000 spectrophotometer (NanoDrop Technologies, Rockland, DE).

\section{2-Gene Multiplex Expression Assay}

Expression of 22 genes was quantified simultaneously in each RNA sample using a custom multiplex gene expression assay, based on traditional reverse transcription-PCR, developed for the Genome Lab GeXP Genetic Analysis System and GeXP Start Kit (Beckman Coulter, Inc., Brea, CA). Genes of interest are summarized in Table 1, including 4 potential reference genes for normalization: ATP synthase $(\boldsymbol{A T P} \boldsymbol{T} \boldsymbol{B})$, hypoxanthine guanine phosphoribosyl transferase 1 (HPRT1), GAPDH, and $\beta-2$ microglobulin (B2M). Primer selection was conducted using eXpress Designer software (Beckman Coulter, Inc.) and GenBank accession numbers (http://www.ncbi.nlm.nih.gov/) of bovine transcripts of interest. In each case, we confirmed that the primers used for each gene produced a single product of the expected size as measured by capillary electrophoresis. Primers were evaluated in both singlet and multiplex reactions to verify that the same amplicons appeared (at the same sizes) in the electropherogram in the multiplex reaction as they appeared in the singlet 
reactions, and that no extraneous bands appeared due to unexpected interaction of the pooled, multiplexed primers.

Procedures for assay optimization, generation of standard curves, reverse transcription, PCR, and capillary electrophoresis on the GeXP Genetic Analysis System were performed as recommended by the manufacturer (GenomeLab GeXP Protocol A29143 AB; December 2007). Additional details on the methodology can be found in Rai et al. (2009). Briefly, $100 \mathrm{ng}$ of total RNA was used in each $20-\mu \mathrm{L}$ reverse transcription reaction containing $20 \mathrm{U}$ of reverse transcriptase (provided), 1,000 pg of KanR RNA (internal control provided with the GeXP start kit), $1 \times$ reverse transcription buffer, 2 $\mu \mathrm{L}$ of pooled gene-specific reverse primers (concentrations optimized according to manufacturer's guidelines), and reaction conditions consisting of $1 \mathrm{~min}$ at $48^{\circ} \mathrm{C}, 60$ min at $42^{\circ} \mathrm{C}$, and $5 \mathrm{~min}$ at $95^{\circ} \mathrm{C}$. A no-template (minus RNA) control reaction was performed with each assay to ensure no contamination of reagents, and a negative control in the absence of reverse transcriptase was performed for each RNA sample to ensure no genomic DNA contamination. Amplification by PCR was performed in duplicate using $9.3 \mu \mathrm{L}$ of $\mathrm{cDNA}, 1 \times \mathrm{PCR}$ buffer, pooled forward primers (at $20 \mathrm{n} M$ each), and 3.5 $\mathrm{U}$ of Thermo-Start DNA polymerase (ABgene, Surrey, UK) under the following conditions: $10 \mathrm{~min}$ at $95^{\circ} \mathrm{C}$, followed by 35 cycles of $30 \mathrm{~s}$ at $94^{\circ} \mathrm{C}, 30 \mathrm{~s}$ at $55^{\circ} \mathrm{C}$, and $1 \mathrm{~min}$ at $68^{\circ} \mathrm{C}$. Resulting PCR products were diluted in sample loading solution and size standard provided in the GeXP kit and separated by capillary electrophoreses in the GeXP Genetic Analysis System using the Frag-3 protocol. Standard curves were generated for each gene in the 22-gene multiplex using a 10-point standard curve ranging from 1 to $500 \mathrm{ng}$ of template RNA.

Results including the peak height and area under the curve for each fragment in the electropherograms were exported to the eXpress Profiler program (Beckman Coulter, Inc.) for analysis and normalization to the internal control (KanR). Stability of 4 potential genes for normalization was assessed using the GeNorm program (http://medgen.ugent.be/ jvdesomp/genorm/) and the most stably expressed genes were used for each comparison of interest (Table 2). Interpolation from the standard curves and final normalization to reference genes were performed using the GeneQuant Tool (Beckman Coulter, Inc.). Thus, normalized expression of each gene transcript was reported as the quantity relative to the geometric mean expression of the selected reference genes.

\section{Immunohistochemistry}

Formalin-fixed bovine GIT tissues were dehydrated in ethanol and embedded in paraffin for subsequent sectioning and staining. Tissues were serially sectioned and mounted onto Superfrost glass slides by Histoserv Inc. (Gaithersburg, MD). The slides were processed for GLP2R protein detection by use of a rabbit polyclonal antibody (MBL Intl., Woburn, MA) raised against human and porcine amino acid sequences. A section of porcine duodenum collected from a 1-yr-old, first-parity gilt at $110 \mathrm{~d}$ of gestation at slaughter was also used as a positive and negative control for the staining procedure. Briefly, slides were deparaffinized and hydrated through sequential alcohol washes. After quenching in $3 \%$ hydrogen peroxide, antigen retrieval was performed by microwaving in $10 \mathrm{mM}$ citrate buffer ( $\mathrm{pH}$ 6.0) for $2 \times 5$-min pulses with a 5-min rest period between each pulse period, and a final 30-min cooling period. Nonspecific binding was blocked by incubating in CAS block (Invitrogen) for $10 \mathrm{~min}$ at room temperature. The GLP2R antibody was applied at a 1:500 dilution in CAS block and incubated at room temperature for $2 \mathrm{~h}$. A negative control was also performed on tissue from the rectum of 1 animal in the absence of GLP2R antibody. The presence of GLP2R was visualized with a 4-min incubation with diaminobenzidine (DAB) using the Super Picture HRP Polymer Conjugate Broad Spectrum staining kit according to the manufacturer's instructions (Invitrogen). Finally, slides were counterstained with Carrazi's hematoxylin for $1 \mathrm{~min}$, dehydrated, and mounted with Permaslip (Alban Scientific Inc., St. Louis, MO).

\section{Statistical Analyses}

For nondetectable gene expression values, a random number between 0 and the lowest detectable value measured among the samples for that particular transcript was assigned to obtain a mean and variance for mean comparisons. Mean comparisons of gene expression across tissues or developmental stages were made by ANOVA using the GLM procedure in SAS (SAS Institute Inc., Cary, NC). For analysis of overall mRNA expression differences across tissues for GCG, PCSK1, $G L P 2 R$, and DPP4, tissue was the CLASS variable and the MODEL statement included each gene as a dependent variable and tissue as the independent effect. To evaluate differential mRNA expression across developmental and lactational stages, tissue and stage were the CLASS variables and the MODEL statement included each gene as a dependent variable and tissue, developmental stage, and their interaction as the independent effects. Further pairwise comparisons of least squares means across tissues or developmental stages within each gene were performed with SAS using the PDIFF option in the LSMEANS statement for each CLASS variable. To determine if expression of 
Gene symbol
Glucagon-like peptide
2 (GLP2) activity

Primer Primer sequence $\left(5^{\prime} \rightarrow 3^{\prime}\right)$ including $5^{\prime}$ adapter sequence

Gene name

Linearity of

tandard

accession no.

curve

$G C G$

GLP2R

PCSK1

DPP 4

Normalization

ATP $5 B$

HPRT1

GAPDH

B2M

Transporter

SGLT1

GLUT2

SLC14A1

PEPT1

Apoptosis

CASP3

CASP6

Proliferation

SOX9

$P C N A$

CCND1

IGF1

AGGTGACACTATAGAATAGCCTTTACTTTGTGGCTGGA GTACGACTCACTATAGGGAATTCATCAACCACTGCACGA AGGTGACACTATAGAATACTCTCCATCGTCTCGCTCTT GTACGACTCACTATAGGGATGAGCAGCCACGAGTAATTG AGGTGACACTATAGAATATGGAAGCAAATCCCAATCTC GTACGACTCACTATAGGGATCGACTGTTCACCATCAAGC AGGTGACACTATAGAATACAGGCCCCTGTAGTCAAAAA GTACGACTCACTATAGGGAGGTAACCACTTCCTCTGCCA

AGGTGACACTATAGAATACCCTCAAGGAGACCATCAAA GTACGACTCACTATAGGGAGGACACCATGGAGGATGAGT AGGTGACACTATAGAATAGCCGACCTGTTGGATTACAT GTACGACTCACTATAGGGAGCATTGTCTTCCCAGTGTCA AGGTGACACTATAGAATAGATTGTCAGCAATGCCTCCT GTACGACTCACTATAGGGACCATCCACAGTCTTCTGGGT AGGTGACACTATAGAATAAGCGTCCTCCAAAGATTCAA GTACGACTCACTATAGGGAACAGGTCTGACTGCTCCGAT

AGGTGACACTATAGAATAACACAGACACCTTGCAGACG GTACGACTCACTATAGGGAAGATGATGGTCAGCCCAAAG AGGTGACACTATAGAATATTGGTGTGATCAATGCACCT GTACGACTCACTATAGGGAAGACAGGGACCAGAACATGG AGGTGACACTATAGAATACTTGAACTCCGTGTTGAGCA GTACGACTCACTATAGGGAGATCAAGGTGCTTGGGAAAA AGGTGACACTATAGAATAAGCAAAGGCTTTCGAAGACA

AGGTGACACTATAGAATAAATGCAACTCTTCCACCAGC GTACGACTCACTATAGGGACTGTGAAGAATCTAGCCCGC AGGTGACACTATAGAATATGCTAGCCACGTAGATGCAG GTACGACTCACTATAGGGACGACACGCCTGAATGATAAA

AGGTGACACTATAGAATAAATCTCCTGGACCCCTTCAT GTACGACTCACTATAGGGATCGCTCTCCTTCTTCAGGTC AGGTGACACTATAGAATAAGGACAGTGCCTTCATTTGG GTACGACTCACTATAGGGAAAACACTGCCCTACAACGCT GTACGACTCACTATAGGGAAAACACTGCCCTACAACGCT AGGTGACACTATAGAATACATGAACTACCTGGACCGCT GTACGACTCACTATAGGGAGCATCTTGGAGAGGAAGTGC AGGTGACACTATAGAATAGTTGGTGGATGCTCTCCAGT GTACGACTCACTATAGGGACCTCCTCAGATCACAGCTCC

Enzyme activity

$M G A M$

ANPEP
AGGTGACACTATAGAATACGAAGAACGTGGCTGCTTAT GTACGACTCACTATAGGGAACCTCCACACGAAGAGACGA AGGTGACACTATAGAATACAGTACCGCCACAACATGAC GTACGACTCACTATAGGGAATTTCCCAGGAACACATAGAAGTC AGGTGACACTATAGAATAGCCCATGTTCACTCCACTCT GTACGACTCACTATAGGGACCTGAGATCAGACCCAGAGC
Pre-pro glucagon

NM 173916

0.9977

GLP2 receptor

XM_589370

0.9995

Prohormone convertase 1

NM_174412

0.9995

Dipeptidyl peptidase IV

NM_174039

0.9998

ATP synthase

NM 175796

0.9992

Hypoxanthine guanine

phosphoribosyl transferase 1

NM_001034035

0.9985

Glyceraldehyde-3-phosphate

dehydrogenase

NM_001034034 0.9994

NM_173893

0.9995

Sodium-glucose cotransporter

NM_174606

0.9984

Facilitated glucose transporter

NM_001103222

0.9989

Urea transporter

NM_001008666

0.9998

Oligopeptide transporter

NM_001099378

0.9988

Caspase 3

XM_001256573

0.9990

Caspase 6

NM_001035419

0.9994

Transcription factor SOX9

AF278703

0.9938

Proliferating cell nuclear antigen

NM_001034494

0.9995

Cyclin D1

NM_001046273

0.9987

Insulin-like growth factor 1

NM_001077828

0.9996

Sucrase-isomaltase

NM_001114189

0.9994

Maltase-glucoamylase

XM_594452

0.9991

Aminopeptidase

NM 001075144

0.9996 
$G C G$ or GLP2R might influence apoptosis, proliferation, growth, enzyme activity, and blood flow in GIT, Pearson correlation coefficients were determined for $G C G$ and GLP2R mRNA versus mRNA expression of molecular markers for the processes of interest, using normalized expression values obtained across all developmental stages in each GIT tissue. A $P$-value of 0.05 was considered significant for all statistical inferences excluding correlation analyses where a $P$-value of 0.01 was used.

\section{RESULTS}

\section{Quality Controls}

Quality of total RNA as assessed by the Agilent RNA Integrity Number (RIN) ranged from 5.0 to 9.7 , and averaged 8.1. A total of 3 (2 omasum and 1 rumen) out of 135 samples had a RIN value $<6$. As a point of reference, a RIN value of $\geq 5$ is recommended for downstream applications such as quantitative PCR (Fleige and Pfaffl, 2006). Linearity of the standard curves for the 22 genes in the multiplex ranged from 0.9938 to 0.9998 (Table 1). Negative control reactions for multiplex reverse transcription-PCR resulted in no amplification of target transcripts (data not shown).

\section{GLP-2 Pathway Gene Expression}

Relative mRNA expression of GLP-2 pathway members $G C G, P C S K 1, D P P 4$, and $G L P 2 R$ varied $(P<$ 0.001; Figure 1) across the 9 GIT tissues collected from prepubertal and mature dairy cattle. Expression of transcripts for GCG and PCSK1 was below the reliable detection limit in rumen, reticulum, and omasum. Expression of DPP4 and GLP2R mRNA was either nondetectable or extremely low in these 3 tissues. Expression of $G C G$ and $P C S K 1 \mathrm{mRNA}$ was greatest in rectum and expression of $D P P 4$ and $G L P 2 R$ was greatest in small intestine. Expression of GLP2R mRNA was moderately high in cecum relative to other tissues despite low expression levels of other GLP-2 pathway members in this tissue.

For mRNA expression of GLP-2 pathway members, an interaction was found between tissue and developmental stage $(P<0.05)$. Within each tissue type, expression of $G C G \mathrm{mRNA}$ and PCSK1 mRNA did not differ by developmental stage or stage of lactation $(P \geq$ $0.15)$. However, mRNA expression of DPP 4 and GLPQR was affected by developmental stage or lactational state in some tissues (Figure 2). Specifically, in duodenum, $D P P 4 \mathrm{mRNA}$ expression was generally lowest in prepubertal heifers $(P<0.03)$, greatest in nonlactating cows $(P<0.04)$, and intermediate among lactating cows. In 
Table 2. Stability of normalization gene expression in bovine gastrointestinal tract tissues based on GeNorm (http://medgen.ugent.be/ jvdesomp/genorm/)

\begin{tabular}{|c|c|c|c|}
\hline Tissue & $\begin{array}{l}\text { Normalization } \\
\text { genes, }{ }^{1} \mathrm{n}\end{array}$ & $\begin{array}{l}\text { Normalization } \\
\text { genes }^{2}\end{array}$ & $\begin{array}{l}\text { Respective } \\
\text { M values }\end{array}$ \\
\hline All tissues & 3 & $A T P 5 B, G A P D H, H P R T 1$ & $0.455,0.518,0.563$ \\
\hline Rumen & 2 & $A T P 5 B, H P R T 1$ & $0.095,0.095$ \\
\hline Reticulum & 2 & ATP $5 B, H P R T 1$ & $0.180,0.180$ \\
\hline Omasum & 2 & $A T P 5 B, H P R T 1$ & $0.298,0.298$ \\
\hline Abomasum & 4 & $A T P 5 B, B 2 M, G A P D H, H P R T 1$ & $0.551,0.757,0.573,0.664$ \\
\hline Duodenum & 2 & $A T P 5 B, G A P D H$ & $0.227,0.227$ \\
\hline Jejunum & 2 & $A T P 5 B, G A P D H$ & $0.277,0.277$ \\
\hline Ileum & 2 & $A T P 5 B, B 2 M$ & $0.290,0.290$ \\
\hline Cecum & 2 & $A T P 5 B, G A P D H$ & $0.099,0.099$ \\
\hline Rectum & 2 & ATP $5 B, B 2 M$ & $0.338,0.338$ \\
\hline
\end{tabular}

${ }^{1}$ Optimal number based on GeNorm pairwise variations between 2 sequential normalization factors containing an increasing number of genes. A cut-off value of 0.15 was used, below which inclusion of no additional reference genes was determined.

${ }^{2} A T P 5 B=$ ATP synthase HPRT1 = hypoxanthine guanine phosphoribosyl transferase $1 ; B 2 M=\beta-2$ microglobulin.

${ }^{3}$ Stability measure from GeNorm based on average pairwise variation in that gene versus all other normalization genes. A value $<1.5$ is considered acceptable. The lower the $\mathrm{M}$ value for a gene, the more stable its expression.

cecum, $D P P 4 \mathrm{mRNA}$ was greatest in nonlactating cows relative to all other stages $(P \leq 0.05)$. In rectum, $D P P 4$ mRNA expression was greatest in late lactation relative to all other stages of lactation or prepubertal heifers $(P \leq 0.02)$. Expression of $D P P 4$ mRNA in rectum was similar between cows in late lactation and nonlactating cows $(P=0.06)$.

Expression of GLP2R mRNA varied across developmental or lactational stages in several tissues (Figure 2). In duodenum, jejunum, ileum, and rectum, expression of GLP2R mRNA had a similar pattern of expression across developmental stages and was generally lowest in prepubertal heifers relative to all other stages examined.

\section{Correlation of GCG and GLP2R mRNA Expression with Other Molecular Markers}

A significant positive correlation was observed in ileum between $G C G$ mRNA expression and mRNA expression of urea transporter $(S L C 14 A 1 ; \mathrm{r}=0.74$, $P=0.001)$, maltase-glucoamylase $(\boldsymbol{M G A M} ; \mathrm{r}=0.76$, $P=0.001)$, and aminopeptidase ( $\boldsymbol{A N P \boldsymbol { E P }} ; \mathrm{r}=0.65$, $P=0.009)$. In cecum, abundances of $G C G$ mRNA and $I G F-I$ mRNA were positively correlated $(\mathrm{r}=0.62, P$ $=0.01)$. Likewise, in duodenum, a positive correlation was found between $G C G \mathrm{mRNA}$ and sucrose-isomaltase ( $\boldsymbol{S I})$ mRNA $(\mathrm{r}=0.79, P<0.001)$. Last, in jejunum, expression of $G C G$ transcripts was positively correlated with mRNA expression of proliferative markers transcription factor SOX9 (SOXg; r $=0.91, P<0.0001)$ and proliferating cell nuclear antigen $(\boldsymbol{P C N A} ; \mathrm{r}=$ $0.71, P=0.003)$.
Expression of GLP2R mRNA was correlated with expression of both apoptotic and proliferative markers in small intestine and cecum. Specifically, GLP2R mRNA was positively correlated with caspase 3 ( $\boldsymbol{C A S P 3})$ mRNA ( $\mathrm{r}=0.64, P=0.01)$ in cecum and negatively correlated with caspase $6(\boldsymbol{C A S P} \boldsymbol{S}) \mathrm{mRNA}$ in jejunum $(\mathrm{r}=-0.67, P=0.006)$ and cecum $(\mathrm{r}=-0.61, P$ $=0.01)$. Transcripts for proliferative markers PCNA, $I G F 1$, and cyclin D1 ( $C \boldsymbol{C N D} 1)$ were positively correlated with GLP2R mRNA in multiple segments of the small intestine and cecum, with the exception of transcripts of $I G F 1$, which was not correlated to GLP2R in cecum. This included $P C N A$ in duodenum $(\mathrm{r}=0.63, P$ $=0.01)$, jejunum $(\mathrm{r}=0.62, P=0.01)$, and cecum $(\mathrm{r}=$ $0.78, P<0.001) ; C C N D 1$ in duodenum $(\mathrm{r}=0.73, P=$ $0.003)$, jejunum $(\mathrm{r}=0.82, P<0.001)$, ileum $(\mathrm{r}=0.89$, $P<0.0001)$, and cecum $(\mathrm{r}=0.71, P=0.003)$; and $I G F 1$ in duodenum $(\mathrm{r}=0.80, P=0.001)$ and jejunum $(\mathrm{r}=0.76, P<0.001)$. Finally, levels of GLP2R mRNA were positively correlated with nitric oxide synthase 3 (NOS3) mRNA, a marker of blood flow, in all 3 segments of the small intestine (duodenum: $\mathrm{r}=0.85$, jejunum: $\mathrm{r}=0.79$, and ileum: $\mathrm{r}=0.76 ; P<0.001)$.

\section{GLP2R Protein Expression}

Expression of GLP2R protein was examined and detected by immunohistochemistry in duodenum, jejunum, ileum, cecum, and rectum of dairy cattle (Figure 3, panels $\mathrm{A}$ to $\mathrm{E}$ ) and duodenum of pig as a positive control (Figure 3, panel G). There was no staining in the negative control samples for either bovine or porcine tissues (Figure 3, panels $\mathrm{F}$ and $\mathrm{H}$ ) incubated without GLP2R antibody. In each tissue, expression of 

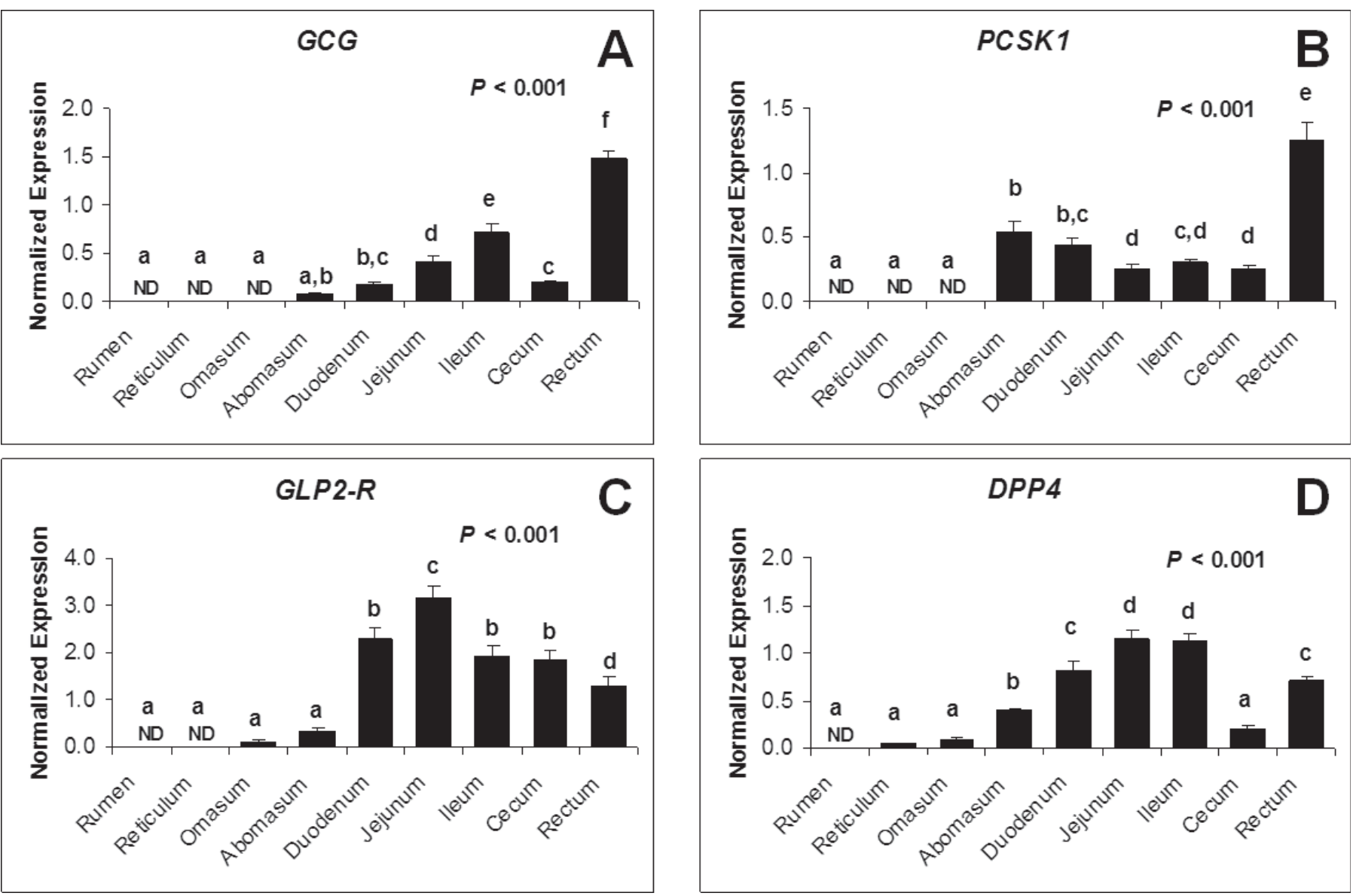

Figure 1. Differential mRNA expression of proglucagon ( $G C G$; panel A), prohormone convertase 1 (PCSK1; panel B), glucagon-like peptide 2 receptor $(G L P 2 R$; panel C), and dipeptidyl peptidase IV (DPP4; panel D) in gastrointestinal tract segments of dairy cattle $(\mathrm{n}=13$ to 15 per tissue). Developmental stages surveyed include prepubertal heifers and lactating and nonlactating cows. ND = less than the reliable detection level. Bars sharing a common letter are not statistically different $(P>0.05)$ from one another.

the protein was limited almost exclusively to cells lining the crypts.

\section{DISCUSSION}

The GLP-2 pathway plays an important role in regulating proliferation and integrity of the intestinal tract of mammals (Estall and Drucker, 2003) and may be involved in intestinal development (Lovshin et al., 2000). Despite the roles of GLP-2 in normal gut function in monogastric species, its functional role in ruminant species has not been well investigated and is poorly understood. The goal of this study was to determine the distribution of the gene expression of the key GLP-2 pathway members, GCG, PCSK1, GLP2R, and DPP4, throughout the bovine GIT using a multiplex gene expression profiling assay, and to determine whether expression of these genes varies among different stages of lactation and development. In addition, we sought to evaluate a putative role of GLP-2 in physiological responses in the gut such as cell proliferation, apoptosis, enzyme activity, nutrient transport, and blood flow by evaluating the relationships between mRNA expression of $G C G$ or GLP2R in GIT tissues and the expression of molecular markers of these processes. Although this is not a direct measure of GLP-2 action on intestinal cells, it provides a basis for inferring a physiological role of GLP-2 in normal bovine GIT function and further investigation into the use of GLP-2 to improve gut function and nutrient uptake in cattle. Results of this study will further provide the basis for examination of gene expression changes in GIT tissues of cattle treated with GLP-2 in future studies.

In the present study, the distribution of $G C G$, PCSK1, GLP2R, and DPP 4 mRNA was characterized in 9 segments of the GIT of dairy cattle and expression was found primarily in the small and large intestines, although expression of all 4 transcripts was also detectable in abomasum. In addition, GLP2R mRNA was detectable in omasum and DPP 4 was detectable in reticulum and omasum of the ruminant GIT. Similarly, expression of $G C G$ mRNA among monogastric species 

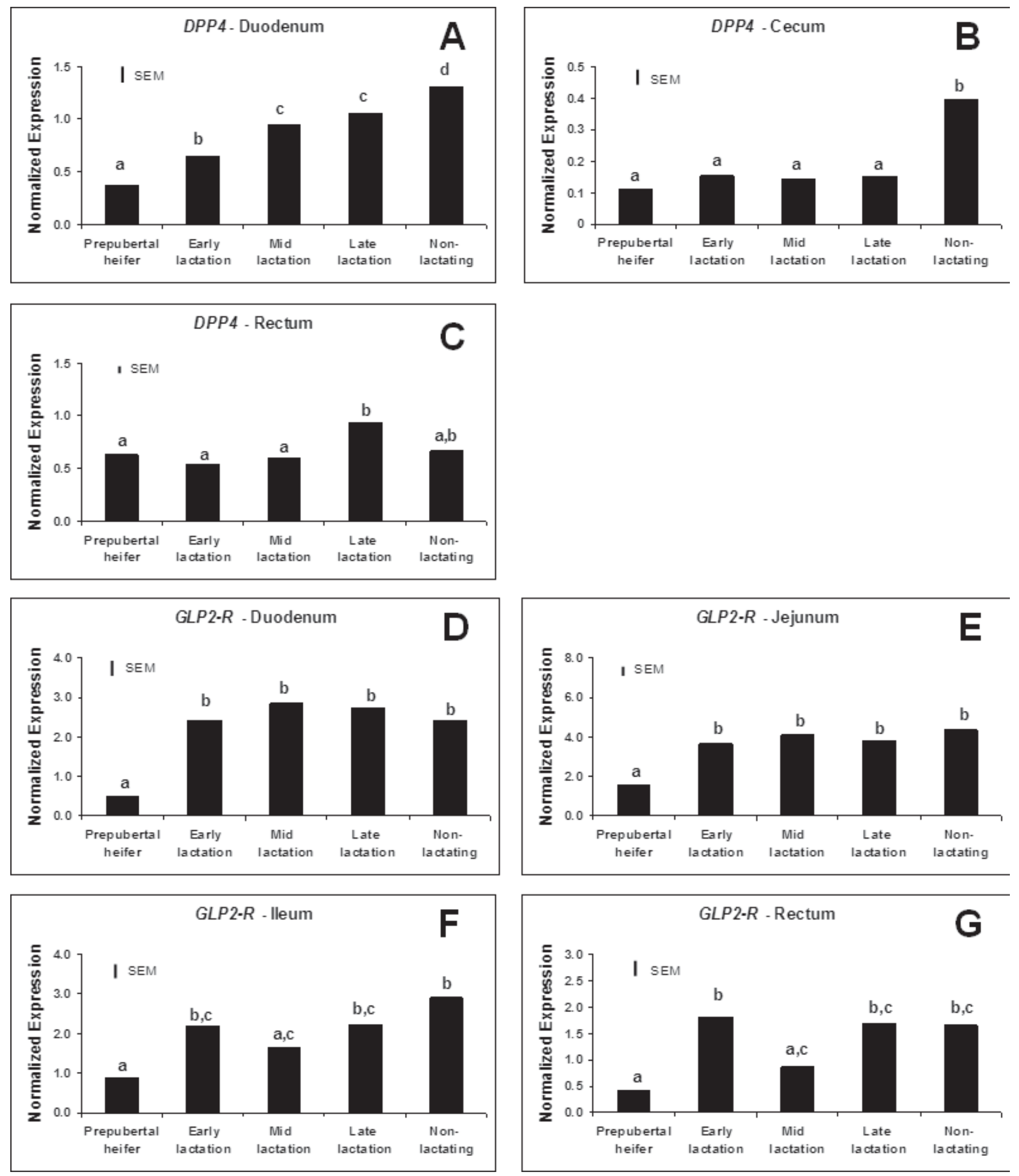

Figure 2. Differential mRNA expression of dipeptidyl peptidase IV ( $D P P 4)$ and glucagon-like peptide 2 receptor $(G L P 2 R)$ across developmental and lactational stages in specific gastrointestinal tract tissues of dairy cattle $(\mathrm{n}=2$ to 3 per stage). The mRNA expression of $D P P 4$ in duodenum (panel A), cecum (panel B), and rectum (panel C); GLP2R mRNA expression in duodenum (panel D), jejunum (panel E), ileum (panel F), and rectum (panel G). Bars sharing a common letter are not statistically different $(P>0.05)$ from one another. SEM $=$ standard error of the mean as determined by ANOVA. 

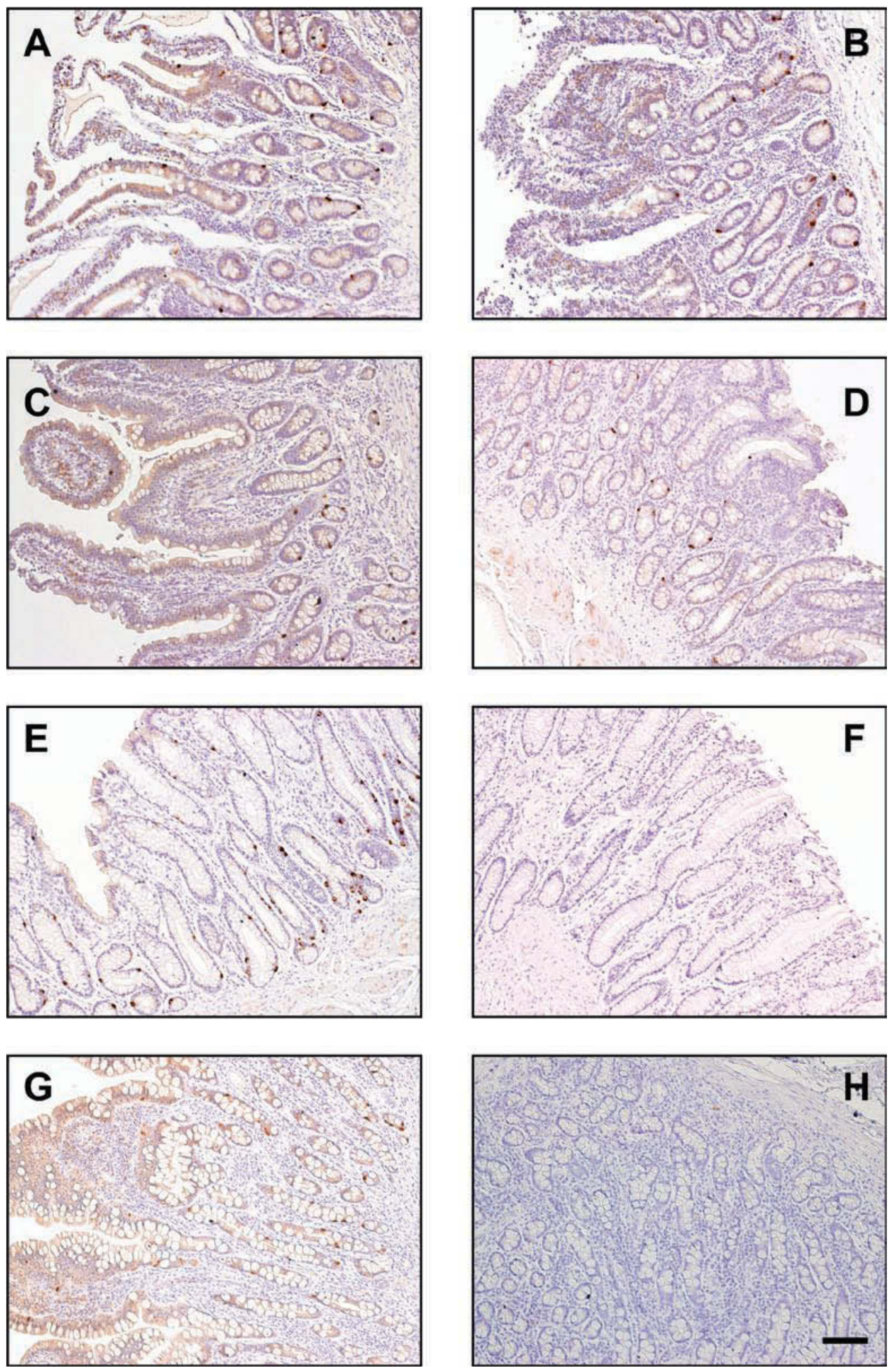

Figure 3. Immunohistochemical localization of glucagon-like peptide 2 receptor (GLP2R) in bovine gastrointestinal tract tissues where GLP2R mRNA was detected by a multiplex gene expression profiling assay. Representative micrographs are shown for (A) duodenum of nonlactating cow; (B) jejunum of mid-lactation cow (d 145); (C) ileum of nonlactating cow; (D) cecum of late-lactation cow (d 250): (E) rectum of early-lactation cow (d 50); (F) negative control [rectum of late-lactation cow (414 d)]; (G) positive control (duodenum of 1-yr-old, first-parity gilt at $110 \mathrm{~d}$ of gestation); and (H) negative control (duodenum of gilt). Negative control slides were incubated without GLP2R antibody and showed no staining. Scale bar $=100 \mu \mathrm{m}$. 
has been demonstrated in the 3 segments of the small intestine, cecum, and colon of the rat (Lovshin et al., 2000; Zhou et al., 2006), and expression in stomach (equivalent to the ruminant abomasum) has been inferred from an expressed sequence tag from a human stomach library (GenBank accession no. BM836042.1). Furthermore, expression of PCSK1 mRNA observed in the present study was found to have a similar tissue distribution to that reported for PCSK1 protein in small intestine of the mouse (Gagnon et al., 2009), and PCSK1 mRNA has been demonstrated in rat stomach (Macro et al., 1996) and colon (Cani et al., 2005), although expression of PCSK1 mRNA has not been reported specifically in cecum. Likewise, the expression pattern of GLP2R mRNA in cattle was consistent with what has been reported in the literature for the rat (Munroe et al., 1999), and protein expression as determined by immunohistochemistry in small intestine, cecum, and rectum of cattle was limited to the cells lining the crypts. This finding agrees with the localization of this $\mathrm{G}$ protein-coupled receptor in human intestine (Yusta et al., 2000). Last, expression of DPP 4 mRNA or its protein has been demonstrated in rat intestine (Dunphy et al., 1999) and stomach (Hong et al., 1989) as well as human small intestine (Darmoul et al., 1994), cecum, and colon (Young et al., 1992), which is consistent with its observed distribution in the bovine GIT. Thus, based on the distribution of mRNA expression of the GLP-2 pathway members and GLP2R protein expression in bovine GIT, it can be inferred that GLP2 has similar functions in ruminants as in monogastric species.

In support of a potential role of GLP-2 in proliferation and maintenance of the ruminant GIT, positive correlations between mRNA expression of the GLP-2 precursor, $G C G$, as well as $G L P 2 R$, were observed between markers of cell proliferation, including transcripts for IGF1, SOX9, PCNA, and CCND1, in multiple segments of the small and large intestines. In addition, a positive correlation was found between $C A S P 3$ and $G L P 2 R$ mRNA in cecum and a negative correlation was observed between $C A S P 6$ mRNA and GLP2R mRNA in duodenum. The relationships with these markers of apoptosis suggest that GLP-2 may mediate apoptosis in these tissues. Burrin et al. (2005) found that GLP-2 treatment of neonatal pigs on total parenteral nutrition dose-dependently decreased CASP6 and CASP3 activities in intestinal tissue, reducing the apoptotic rate of crypt and villus cells, but increased CASP3 protein abundance in intestinal cells. This illustrates the potential disconnect between mRNA abundance and the abundance or activity of its encoded enzyme, and the caution one must exercise when interpreting the physiological implications of changes in mRNA expression. Despite this limitation, the relationships observed between $G C G$ or GLP2R gene expression in bovine GIT and expression of markers of proliferation and apoptosis suggest that GLP-2 may play a role in these processes in cattle and other ruminants.

As a result of reports of GLP-2 effects on intestinal transcripts for enzymes such as glycoside hydrolases and aminopeptidases in piglets (Petersen et al., 2002), relationships between $G C G$ and GLP2R mRNA expression and expression of SI, MGAM, and ANPEP mRNA in bovine GIT were evaluated in the present study. Our results supported a potential role of the GLP-2 pathway in nutrient uptake in ruminant GIT, because expression of $G C G$ was positively correlated with changes in expression of MGAM, ANPEP, and SI genes in bovine small intestine. However, no apparent association was observed between GLP2R mRNA and expression of markers of enzyme activity.

Conversely, a positive correlation between GLP2R and NOS3 mRNA in all 3 segments of the bovine small intestine is consistent with observed effects of GLP-2 infusion in piglets (Burrin et al., 2005). Experimental evidence indicates that GLP-2 stimulates blood flow in GIT through the GLP2R and endothelial nitric oxide synthase activation (Guan et al., 2003, 2006). A preliminary report by Taylor-Edwards et al. (2009a) demonstrated increased splanchnic blood flow in calves infused with GLP-2. It is thought that increased blood flow to gut tissues induced by GLP-2 might be mediated by NOS3 and may contribute to improved nutrient absorption in the ruminant gut.

Specifically, improved intestinal transport and absorption of nutrients including hexoses, amino acids, and lipids have been reported in response to GLP-2 treatment (Burrin et al., 2003; Estall and Drucker, 2006). The exact mechanisms for these responses are not well understood, but may be mediated by changes in the expression of transporters for these nutrients. For instance, Ramsanahie et al. (2004) observed increased expression of sodium-dependent glucose transporter (SGLT1) protein in enterocytes and localized changes in $S G L T 1$ mRNA expression to the intestinal villus of GLP-2-treated rats, and GLP-2 treatment was found to increase abundance of $S G L T 1 \mathrm{mRNA}$ in small intestine of piglets (Sangild et al., 2006). Further, increased expression of the facilitated glucose transporter (GLUT2) protein in the brush border membrane has been observed in GLP-2-treated rats (Au et al., 2002). However, no apparent relationship was observed between expression of $G C G$ or GLP2R mRNA and abundance of SGLT1 or GLUT2 mRNA transcripts in any segments of the bovine GIT. A weak association $(\mathrm{r}=0.63, P=0.02)$ was observed between $G C G$ mRNA and PEPT1 mRNA in duodenum. It should be noted that expression of 
GLUT2 mRNA was below the reliable detection level in most segments of the bovine GIT, excluding duodenum and jejunum, and bovine rectum expressed only SGLT1 mRNA of the 4 transporters examined. In addition, the current study was based on basal expression levels of these genes, whereas the data in the literature are based on responses to GLP-2 supplementation. The effects of pharmacological doses of GLP-2 on bovine intestinal transporter mRNA expression are unknown and are currently under investigation in our laboratory. Of interest, however, was a strong positive correlation between $G C G$ mRNA and expression of urea transporter (SLC14A1) in bovine ileum. This is the first known report of this apparent association and is suggestive of GLP-2 effects on nitrogen salvaging via urea transporter expression in the ruminant gut, warranting further investigation.

Finally, evidence suggests that expression and function of GLP-2 pathway members is developmentally regulated. For instance, although GLP2R mRNA is expressed in fetal and neonatal piglets, administration of pharmacological doses of GLP-2 does not induce intestinal proliferation and differentially affects intestinal enzyme activity between fetal and neonatal piglets (Petersen et al., 2001). Furthermore, intestinal GCG mRNA expression significantly changes with age and weaning in rats (Reimer et al., 1997). To evaluate a potential role of the GLP-2 pathway in ruminant GIT development, we examined the distribution of $G C G$, PCSK1, GLP2R, and DPP4 mRNA in prepubertal calves and adult cows during different stages of lactation. Despite a small sample size, differential mRNA expression across the stages examined was observed for DPP 4 and GLP2R. The variation in DPP\& mRNA expression was in duodenum, cecum, and rectum, with expression levels generally greatest in nonlactating cows or cows in late lactation, depending on the tissue examined. Because DPP4 plays a role in cleavage of multiple peptide targets, these changes may be related to functions other than inactivation of GLP-2; however, the increases in DPP $4 \mathrm{mRNA}$ expression would likely result in reduced GLP-2-mediated responses in these tissues because DPP4 appears to be regulated at the transcript level (Hong et al., 1989; Darmoul et al., 1991). In other words, one would expect an increase in intestinal DPP4 mRNA expression to reduce intestinal growth mediated by GLP-2. Darmoul et al. (1994) measured DPP 4 mRNA and its protein expression in human small intestine and colon, as well as DPP4 enzyme activity in epithelial cells isolated from these tissues and found increasing mRNA and protein levels from duodenum to ileum, and nondetectable levels in colon by Northern and Western blotting. Low-level DPP \& mRNA expression could only be detected by the more sensitive technique of reverse
transcription-PCR. Expression of bovine $\mathrm{DPP}_{4}$ mRNA as measured by reverse transcription-PCR in the present study showed a pattern of expression similar to that of human. Interestingly, in a previous study examining changes in intestinal proliferation during 4 stages of lactation in dairy cattle by our laboratory (Baldwin et al., 2004), it was found that mean meter weight of duodenum of lactating dairy cows declined from approximately $275 \mathrm{~g}$ at d 90 of lactation, to $240 \mathrm{~g}$ at d 120 of lactation, and to $234 \mathrm{~g}$ at $240 \mathrm{~d}$ of lactation. This would correspond to measures of gene expression at early, mid, and late lactation in our study in which the mean duodenal $D P P 4 \mathrm{mRNA}$ values had a correlation of -0.99 with the corresponding mean duodenum weights in the study by Baldwin et al. (2004). Although this is highly circumstantial evidence, it is consistent with the hypothesis that DPP4 may play a role in intestinal growth regulation in dairy cattle during lactation.

The basic expression pattern of GLP2R mRNA measured in prepubertal heifers and cows during different stages of lactation in our study showed relatively stable transcript expression in small intestine and rectum in adult cows regardless of lactational stage. However, expression in adult cows was at a greater level than in prepubertal heifers. This would suggest that basal $G L P 2 R$ expression might only be responsive to or mediate relatively large changes in intestinal growth, such as those involved in the transition from a prepubertal state to a pregnant or lactating state, where energy demands and nutrient intake are substantially greater than in the young animal. Clearly, an experiment specifically designed to examine the ontogeny of GLP2R mRNA and GLP2R protein expression during intestinal development in ruminants is needed.

\section{CONCLUSIONS}

Because of the importance of GLP-2 in gut development, proliferation, and integrity in monogastric species, the GLP-2 pathway may contribute to several economically relevant processes in ruminant livestock species. Results of this study suggest that the GLP2 pathway is expressed and functional in the bovine GIT. Understanding mechanisms governing the GLP-2 pathway in cattle may ultimately provide novel means to improve GIT development and function in calves, or manipulate proliferation of the GIT, one of the greatest nutrient users among the visceral tissues (Johnson et al., 1990), in adult dairy cows during lactation and the dry period.

\section{ACKNOWLEDGMENTS}

We thank Dennis Hucht, Mary Niland, Marsha Campbell, Mary Bowman, Sarah Rodriguez-Hazelton, 
and Research Support Services staff at the USDA-ARS (Beltsville, MD) for excellent technical assistance. Thanks also to Stanislaw Kahl (USDA-ARS, Beltsville, MD) for assistance with statistical analysis. Mention of trade names or commercial products in this article is solely for the purpose of providing specific information and does not imply recommendation or endorsement by the US Department of Agriculture.

\section{REFERENCES}

Au, A., A. Gupta, P. Schembri, and C. I. Cheeseman. 2002. Rapid insertion of GLUT2 into the rat jejunal brush-border membrane promoted by glucagon-like peptide 2. Biochem. J. 367:247-254.

Baldwin, R. L., K. R. McLeod, and A. V. Capuco. 2004. Visceral tissue growth and proliferation during the bovine lactation cycle. J. Dairy Sci. 87:2977-2986.

Burrin, D. G., B. Stoll, and X. Guan. 2003. Glucagon-like peptide 2 function in domestic animals. Domest. Anim. Endocrinol. $24: 103-122$.

Burrin, D. G., B. Stoll, X. Guan, L. Cui, X. Chang, and D. Hadsell. 2007. GLP-2 rapidly activates divergent intracellular signaling pathways involved in intestinal cell survival and proliferation in neonatal piglets. Am. J. Physiol. Endocrinol. Metab. 292:E281E291.

Burrin, D. G., B. Stoll, X. Guan, L. Cui, X. Chang, and J. J. Holst. 2005. Glucagon-like peptide 2 dose-dependently activates intestinal cell survival and proliferation in neonatal piglets. Endocrinology $146: 22-32$

Cani, P. D., C. A. Daubioul, B. Reusens, C. Remacle, G. Catillon, and N. M. Delzenne. 2005. Involvement of endogenous glucagon-like peptide 1(7-36) amide on glycaemia-lowering effect of oligofructose in streptozotocin-treated rats. J. Endocrinol. 185:457-465.

Darmoul, D., C. Rouyer-Fessard, A. Blais, T. Voisin, C. Sapin, L. Baricault, C. Cibert, G. Geraud, A. Couvineau, M. Laburthe, and G. Trugnan. 1991. Dipeptidyl peptidase IV expression in rat jejuna crypt-villus axis is controlled at mRNA level. Am. J. Physiol. 261:G763-G769.

Darmoul, D., T. Voisin, A. Couvineau, C. Rouyer-Fessard, R. Salomon, Y. Wang, D. M. Swallow, and M. Laburthe. 1994. Regional expression of epithelial dipeptidyl peptidase IV in the human intestines. Biochem. Biophys. Res. Commun. 203:1224-1229.

Drucker, D. J. 2005. Biologic actions and therapeutic potential of the progulucagon-derived peptides. Nat. Clin. Pract. Endocrinol. Metab. $1: 22-31$.

Dunphy, J. L., F. A. Justice, R. G. Taylor, and P. J. Fuller. 1999. mRNA levels of dipeptidyl peptidase IV decrease during intestinal adaptation. J. Surg. Res. 87:130-133.

Estall, J. L., and D. J. Drucker. 2003. Dual regulation of cell proliferation and survival via activation of glucagon-like pepetide-2 receptor signaling. J. Nutr. 133:3708-3711.

Estall, J. L., and D. J. Drucker. 2006. Glucagon-like peptide-2. Annu. Rev. Nutr. 26:2.1-2.22.

Fleige, S., and M. W. Pfaffl. 2006. RNA integrity and the effect on the real-time qRT-PCR performance. Mol. Aspects Med. 27:126139

Gagnon, J., J. Mayne, M. Mbikay, J. Woulfe, and M. Chrétien. 2009. Expression of PCSK1 (pC1/3), PCSK2 (PC2) and PCSK3 (furin) in mouse small intestine. Regul. Pept. 152:54-60.

Guan, X., H. E. Karpen, J. Stephens, J. T. Bukowski, S. Niu, G. Zhang, B. Stoll, M. J. Finegold, J. J. Holst, D. Hadsell, B. L. Nichols, and D. G. Burrin. 2006. GLP-2 receptor localizes to enteric neurons and endocrine cells expressing vasoactive peptides and mediates increased blood flow. Gastroenterology 130:150-164.

Guan, X. F., B. Stoll, X. F. Lu, K. A. Tappenden, J. J. Holst, B. Hartmann, and D. G. Burrin. 2003. GLP-2-mediated up-regulation of intestinal blood flow and glucose uptake is nitric oxide-dependent in TPN-fed piglets. Gastroenterology 125:136-147.
Hong, W. J., J. K. Petell, D. Swank, J. Sanford, D. C. Hixson, and D. Doyle. 1989. Expression of dipeptidyl peptidase IV in rat tissues is mainly regulated at the mRNA levels. Exp. Cell Res. $182: 256-266$.

Jeppesen, P. B. 2003. Clinical significance of GLP-2 in short-bowel syndrome. J. Nutr. 133:3721-3724.

Johnson, D. E., K. A. Johnson, and R. L. Baldwin. 1990. Changes in liver and gastrointestinal tract energy demands in response to physiological workload in ruminants. J. Nutr. 120:649-655.

Lovshin, J., Y. Bernardo, I. Iliopoulos, A. Migirdicyan, L. Dableh, P. L. Brubaker, and D. J. Drucker. 2000. Ontogeny of the glucagonlike peptide-2 receptor axis in the developing rat intestine. Endocrinology 141:4194-4201.

Lovshin, J., and D. J. Drucker. 2000. New frontiers in the biology of GLP-2. Regul. Pept. 90:27-32.

Macro, J. A., R. Dimaline, and G. J. Dockray. 1996. Identification and expression of prohormone-converting enzymes in the rat stomach. Am. J. Physiol. 270:G87-G93.

Munroe, D. G., A. K. Gupta, F. Kooshesh, T. B. Vyas, G. Rizkalla, H. Wang, L. Demchyshyn, Z. J. Yang, R. K. Kamboj. H. Chen, K. McCallum, M. Sumner-Smith, D. J. Drucker, and A. Crivici. 1999. Prototypic G protein-coupled receptor for the intestinotrophic factor glucagon-like peptide 2. Proc. Natl. Acad. Sci. USA 96:15691573.

Petersen, Y. M., D. G. Burrin, and P. T. Sangild. 2001. GLP-2 has differential effects on small intestine growth and function in fetal and neonatal pigs. Am. J. Physiol. Regul. Integr. Comp. Physiol. 281:R1986-R1993.

Petersen, Y. M., J. Elnif, M. Schmidt, and P. T. Sangild. 2002. Glucagon-like peptide 2 enhances maltase-glucoamylase and sucraseisomaltase gene expression and activity in parenterally fed premature neonatal piglets. Pediatr. Res. 52:498-503.

Rai, A. J., R. M. Kamath, W. Gerald, and M. Fleisher. 2009. Analytical validation of the GeXP analyzer and design of a workflow for cancer-biomarker discovery using multiplexed gene-expression profiling. Anal. Bioanal. Chem. 393:1505-1511.

Ramsanahie, A. P., U. V. Berger, M. J. Zinner, E. E. Whang, D. B. Rhoads, and S. W. Ashley. 2004. Effect of glucagon-like peptide-2 (GLP-2) on diurnal SGLT1 expression. Dig. Dis. Sci. 49:17311737 .

Reimer, R. A., C. J. Field, and M. I. McBurney. 1997. Ontogenic changes in proglucagon mRNA in BB diabetes prone and normal rats weaned onto a chow diet. Diabetologia 40:871-878.

Sangild, P. T., K. A. Tappenden, C. Malo, Y. M. Petersen, J. Elnif, A. L. Bartholome, and R. K. Buddington. 2006. Glucagon-like peptide 2 stimulates intestinal nutrient absorption in parenterally fed newborn pigs. J. Pediatr. Gastroenterol. Nutr. 43:160-167.

Taylor-Edwards, C. C., D. G. Burrin, J. J. Holst, K. R. McLeod, and D. L. Harmon. 2009a. Glucagon-like peptide-2 increases splanchnic blood flow acutely in calves but loses effectiveness with chronic exposure. J. Anim. Sci. 87(E-Suppl. 2):234. (Abstr.)

Taylor-Edwards, C. C., D. G. Burrin, K. R. McLeod, and D. L. Harmon. 2009b. Glucagon-like peptide-2 increases small intestinal mass of calves. J. Anim. Sci. 87(E-Suppl. 2):234. (Abstr.)

Taylor-Edwards, C. C., D. B. Edwards, M. J. Doig, E. S. Vanzant, K. R. McLeod, J. A. Boling, J. C. Matthews, and D. L. Harmon. 2008. Proglucagon and GLP-2 receptor mRNA distribution in the ruminant gastrointestinal tract. J. Anim. Sci. 86(E-Suppl. 2):428. (Abstr.)

Young, G. P., F. A. Macrae, P. R. Gibson, M. Alexeyeff, and R. H. Whitehead. 1992. Brush border hydrolases in normal and neoplastic colonic epithelium. J. Gastroenterol. Hepatol. 7:347-354.

Yusta, B., L. Huang, D. Munroe, G. Wolff, R. Fantaske, S. Sharma, L. Demchyshyn, S. L. Asa, and D. J. Drucker. 2000. Enteroendocrine localization of GLP-2 receptor expression in humans and rodents. Gastroenterology 119:744-755.

Zhou, J., M. Hegsted, K. L. McCutcheon, M. J. Keenan, X. Xi, A M. Raggio, and R. J. Martin. 2006. Peptide YY and proglucagon mRNA expression patterns and regulation in the gut. Obesity (Silver Spring) 14:683-689. 